\title{
Correlation of morphological and radiological characteristics of degenerative disc disease in lumbar spine: a cadaveric study
}

\author{
P.A. Pękala'1, 2,3*, D. Taterra1,2*, K. Krupa1,2, M. Paziewski1,2, W. Wojciechowski4, \\ T. Konopka ${ }^{5}$, J.A. Walocha' ${ }^{1,2}$, K.A. Tomaszewski ${ }^{1,3,6}$ \\ IInternational Evidence-Based Anatomy Working Group, Krakow, Poland \\ ${ }^{2}$ Department of Anatomy, Jagiellonian University Medical College, Krakow, Poland \\ ${ }^{3}$ Faculty of Medicine and Health Sciences, Andrzej Frycz Modrzewski Krakow University, Krakow, Poland \\ ${ }^{4}$ Department of Radiology, Jagiellonian University Medical College, Krakow, Poland \\ ${ }^{5}$ Department of Forensic Medicine, Jagiellonian University Medical College, Krakow, Poland \\ ${ }^{6}$ Scanmed St. Raphael Hospital, Krakow, Poland
}

[Received: 12 January 2021; Accepted: 15 March 2021; Early publication date: 13 April 2021]

Background: Intervertebral disc (IVD) degeneration plays a crucial role in the pathophysiology of low back pain. Several grading systems have been developed for both morphological and radiological assessment. The aim of this study was to assess the morphological and radiological characteristics of IVD degeneration and validate popular radiological Pfirrmann scale against morphological Thompson grading system.

Materials and methods: Full spinal columns (vertebrae L1-S1 and IVD between them) were harvested from cadavers through an anterior dissection. Magnetic resonance imaging scans of all samples were conducted. Then, all vertebral columns were cut in the midsagittal plane and assessed morphologically.

Results: A total of 100 lumbar spine columns (446 IVDs) were included in the analysis of the degeneration grade. Morphologic Thompson scale graded the majority of discs as grade 2 and $3(44.2 \%$ and $32.1 \%$, respectively), followed by grade $4(16.8 \%)$, grade 1 (5.8\%) and grade 5 (1.1\%). The radiologic Pfirrmann grading system classified $44.2 \%$ of discs as grade $2,32.1 \%$ as grade $3,16.8 \%$ as grade $4,5.8 \%$ as grade 1 , and $1.1 \%$ as grade 5 . The analysis on the effect of age on degeneration revealed significant, although moderate, positive correlation with both scales. Analysis of the agreement between scales showed weighted Cohen's kappa equal to $0.61(p<0.001)$. Most of the disagreement occurred due to a 1 grade difference (91.5\%), whereas only $8.5 \%$ due to a 2 grade difference. Conclusions: With the increase of the prevalence of IVD disease in the population, reliable grading systems of IVD degeneration are crucial for spine surgeons in their clinical assessment. While overall there is agreement between both grading systems, clinicians should remain careful when using Pfirrmann scale as the grades tend to deviate from the morphological assessment. (Folia Morphol 2022; 81, 2: 503-509)

Key words: low back pain, discopathy, Thompson scale, Pfirrmann scale

Address for correspondence: P.A. Pękala, MD, PhD, Department of Anatomy, Jagiellonian University Medical College, ul. Kopernika 12, 31-034 Kraków, Poland, tel/fax: +48 1242295 11, e-mail: pekala.pa@gmail.com

*Equal contributors

This article is available in open access under Creative Common Attribution-Non-Commercial-No Derivatives 4.0 International (CC BY-NC-ND 4.0) license, allowing to download articles and share them with others as long as they credit the authors and the publisher, but without permission to change them in any way or use them commercially. 


\section{INTRODUCTION}

Lower back pain (LBP) remains a leading cause of disability and morbidity in today's society. Even up to $70 \%$ of the population experiences LBP throughout their lives [5]. With an estimated societal cost of LBP at 85 billion dollars annually, only in the United States in 2008, and expected several-fold increase in the next decades, it constitutes an enormous burden to the healthcare system [7].

The aetiology of LBP is multifactorial with both genetic and environmental factors contributing to its development. Intervertebral disc (IVD) degeneration is considered to be a crucial component in the aetiology of this condition. The IVD is formed by gelatinous, centrally located nucleus pulposus (NP), which is surrounded by annulus fibrosus (AF). Morphologically, the NP consists of collagen II fibres and elastin randomly arranged in highly hydrated, aggrecan-based gel, which also contains low concentration of chondrocyte-like cells [15]. The AF can be divided into an inner $A F$, which can be viewed as a transition zone and the outer AF, which in turn is formed by distinct, highly organized lamellae consisting of collagen I fibres, intertwined with elastin, lubricin and collagen VI fibres [15]. Moreover, the IVD is bound caudally and rostrally by IVD endplates, which separate intervertebral bodies from the IVD. The highly hydrated NP, which is constrained both by the AF and the endplates, distributes mechanical loads evenly, dissipates energy and allows for the movement of the vertebral column [15]. Deterioration in the function of IVD is associated with the changes in the content of extracellular matrix of the NP, which occur with age and degeneration [10]. These include loss of water content, degradation of proteoglycans and collagen as well as upregulation of inflammatory cytokines [10]. The deterioration of the NP leads to irreversible structural changes of the IVD and its surrounding. A common macroscopic characteristic of degeneration is the presence of clefts and tears within the IVD and the loss of demarcation between the NP and the $A F$ [11]. As such, the IVD loses its mechanical bearing properties [17] with a transfer of pressure exertion point from NP to AF [1]. This can result in NP bulging, herniation, compression syndrome and effectively low back pain.

There are several grading systems used to assess the degree of degeneration based on the modality used. A grading system to assess morphologic changes due to IVD degeneration was proposed by Thomp- son et al. [14] in 1990. Moreover, the magnetic resonance imaging (MRI) has been widely used to study and assess IVD degeneration. The signal intensity loss in T2-weighted images correlates with the degree of degeneration [4]. Pfirrmann et al. [9] classification is a popular grading system for the degenerative changes of the lumbar spine observed in MRI. It is broadly used to study lumbar degeneration, but has also been adopted by neurosurgeons and orthopaedic surgeons in the perioperative setting. It has been validated in multiple studies $[12,16]$; however, the assessment of the correlation between macroscopic Thompson's and radiologic Pfirrmann's grading systems has not been studied comprehensively.

Therefore, the aim of this study was to compare the macroscopic appearance of the lumbar spine specimens with their MRI appearance and check the reliability of the popular Pfirrmann classification of the degenerative changes in the lumbar spine.

\section{MATERIALS AND METHODS}

\section{Specimen collection}

The study protocol was approved by our institutional Bioethics Committee. Moreover, study strictly adhered to ethical principles for medical research involving human subjects set by the Declaration of Helsinki.

Full spinal columns (vertebrae L1-S1 and IVD between them) were harvested from fresh cadavers through an anterior dissection. Inclusion criteria were as follows: 1 ) age $18-80,2$ ) possibility to dissect specific lumbar column. Any donors that were deceased due to trauma or had a visible spinal trauma, spinal surgery, spinal tumours, ankylosing spondylitis were excluded from this study.

Intervertebral discs that became damaged during dissection or had artefacts in MRI scans that did not allow for full and reliable assessment were excluded from further analyses.

\section{Magnetic resonance imaging}

Magnetic resonance imaging scans of the harvested spinal columns were conducted with the use of Philips Achieva 3.0T TX apparatus. Two independent reviewers assessed the IVD degeneration according to Pfirrmann et al. [9] scale. In summary, Pfirrmann grading system assesses changes in T2 spin-echo weighted images on a scale from 1 to 5 , with grade 1 describing healthy disc (homogeneous with bright hyperintense white signal intensity and normal disc 
height), while grade 5 describing heavily degenerated disc (disc space is collapsed, inhomogeneous with a hypointense black signal intensity) (Fig. 1) [9].

Moreover, the MRI scans were assessed for Modic type endplate changes [8]. Type 1 changes were defined as decreased signal intensity on T1-weighted images and increased signal intensity on T2-weighted images. Type 2 changes were defined as increased signal intensity on T1-weighted images and isointense or slightly increased signal intensity on T2-weighted images [8]. Modic type III changes showed decreased signal intensity on both T1- and T2-weighted images. Any radiologic findings, such as Schmorl's nodes, disc bulging or herniation were also noted.

\section{Morphologic assessment}

All vertebral columns were cut in the midsagittal plane. High resolution images of each column were taken and used for later assessment. The IVD degeneration was graded on a scale from 1 to 5 based on criteria developed by Thompson et al. [14] by two independent reviewers. In summary, each grade is determined through assessing specific morphologic changes of nucleus pulposus, annulus fibrosus, IVD end-plates and adjacent vertebral bodies with grade 1 being healthy IVD, while grade 5 being heavily degenerated disc (Fig. 1) [14].

Moreover, any macroscopic alterations in the structure of lumbar columns were noted and included the following: osteophytes, Schmorl's nodes, IVD clefts, tears, bulging and herniation.

\section{Statistical analysis}

All statistical analyses were conducted using STATISTICA (v.13.3) and PQStat (v.1.8.0). Frequency distribution, mean and standard deviation were used to characterise study group and degeneration grades. Spearman's rank correlation coefficient statistic was conducted to assess the relation between the age and degeneration. Moreover, in order to determine agreement between specific Pfirrmann and Thompson grades, weighted Cohen's kappa coefficient was utilised. This statistic assigns weights to disagreement values, with the higher the degree of disagreement the higher the weight. A kappa value of 1 indicates perfect agreement, while value of 0 indicates agreement equivalent to chance. A p-value of $<0.05$ determines statistically significant agreement between the two scores. Subgroup analysis on the agreement between degeneration grades for specific IVD levels was also conducted.

\section{RESULTS}

\section{Study group}

One hundred lumbar spine columns (L1-S1) were harvested from male cadavers. Mean age of the donor was $42.2 \pm 12.3$ years. There were 54 IVDs which visualisation did not allow for full and reliable assessment, therefore authors decided to exclude them from the analysis.

\section{Degeneration assessment}

A total of 446 IVDs were included in the analysis of the degeneration grade. Radiologic assessment using the Pfirrmann grading system classified $44.2 \%$ of discs as grade $2,32.1 \%$ as grade $3,16.8 \%$ as grade 4 , $5.8 \%$ as grade 1 and $1.1 \%$ as grade 5 . Morphologic Thompson scale graded the majority of discs as grade 2 and $3(44.2 \%$ and $32.1 \%$, respectively), followed by grade $4(16.8 \%)$, grade $1(5.8 \%)$ and grade $5(1.1 \%)$.

There were 42 discs ( $9.4 \%$ of all discs) that showed Modic type endplate changes, with $8.7 \%$ of all discs grades as Modic type 2 and $0.7 \%$ as Modic type 1 .

The analysis on the effect of age on degeneration revealed significant, although moderate, positive correlation with both Thompson $(\rho=0.38, \mathrm{p}<0.001)$ and Pfirmann $(\rho=0.36, \mathrm{p}<0.001)$ average grade.

Table 1 summarises subgroup analyses of the Thompson and Pfirrmann grades based on the spinal level.

\section{Inter-grading system agreement}

A total of 446 pairs of Thompson and Pfirrmann grades for specific IVDs were compared. Analysis showed weighted Cohen's kappa equal to 0.61 ( $p<0.001$ ), which suggests significant and substantial agreement between the two grading systems. The highest percentage agreement was achieved for grade 2 ( $67.2 \%$ of discs). All other grades showed an agreement in less than half of the cases. The highest percentage disagreement was observed for Thompson grade 1 with $70.0 \%$ of discs graded as Pfirrmann grade 2. Most of the disagreement occurred due to a 1 grade difference $(91.5 \%)$, whereas only $8.5 \%$ due to a 2 grade difference.

In summary, Pfirrmann scale tended to underscore degeneration when compared to Thompson grades. Majority of Thompson grades 5 were scored as Pfirrmann grades $4-5(83.3 \%)$. Thompson grades 4 

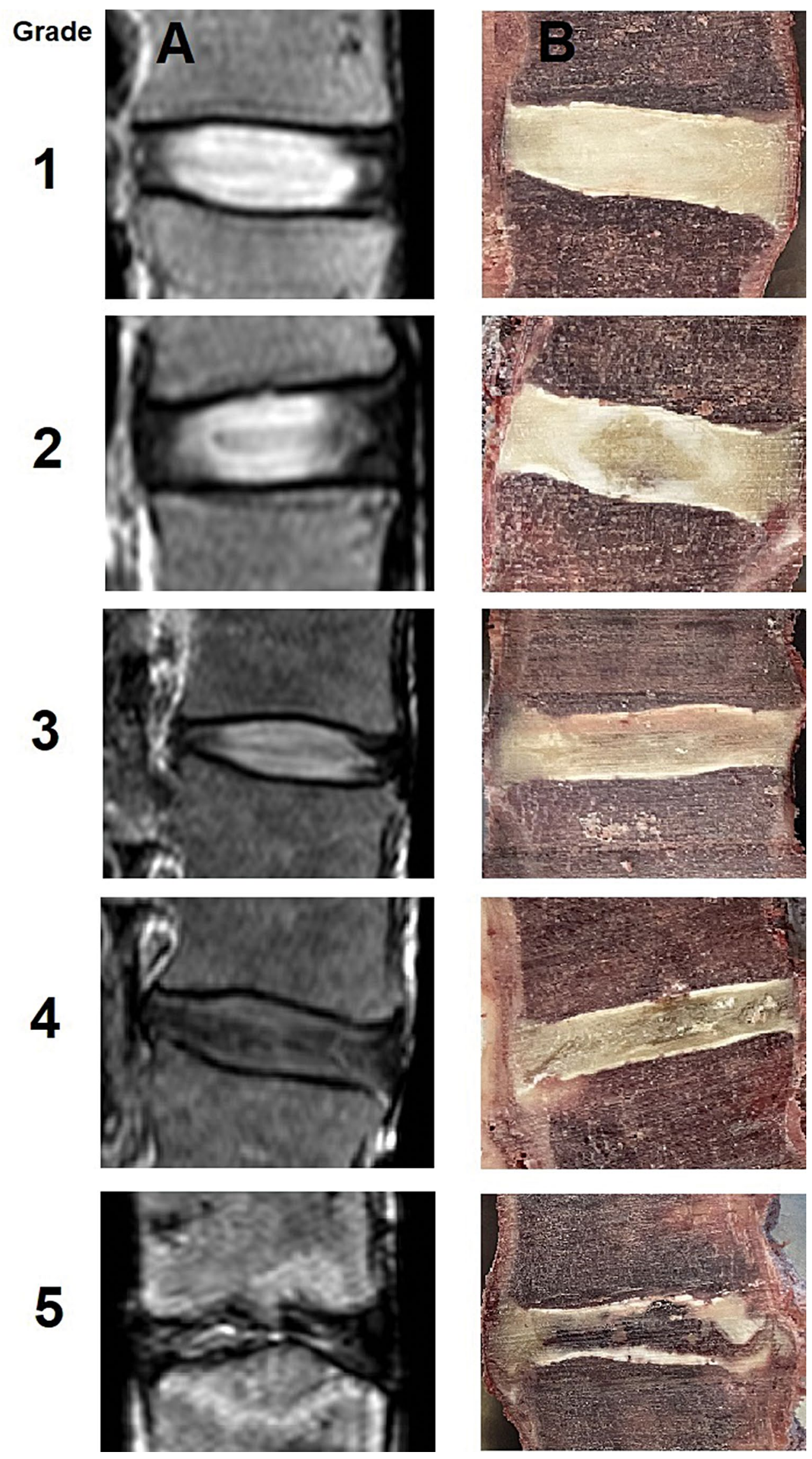

Figure 1. Representative micrographs of radiologic Pfirrmann (A) and morphologic Thompson (B) grading scores. 
Table 1. Subgroup analyses of the Thompson and Pfirmann grades based on the spinal level

\begin{tabular}{|c|c|c|}
\hline Intervertebral disc level & $\begin{array}{c}\text { Thompson } \\
\text { grades }\end{array}$ & $\begin{array}{c}\text { Pfirrmann } \\
\text { grades }\end{array}$ \\
\hline \multirow[t]{5}{*}{ L1/L2 } & Grade $1-7 \%$ & Grade $1-4 \%$ \\
\hline & Grade $2-39 \%$ & Grade $2-63 \%$ \\
\hline & Grade $3-50 \%$ & Grade $3-24 \%$ \\
\hline & Grade $4-4 \%$ & Grade $4-9 \%$ \\
\hline & Grade $5-0 \%$ & Grade $5-0 \%$ \\
\hline \multirow[t]{5}{*}{ L2/L3 } & Grade $1-7 \%$ & Grade $1-3 \%$ \\
\hline & Grade $2-32 \%$ & Grade $2-59 \%$ \\
\hline & Grade $3-50 \%$ & Grade $3-31 \%$ \\
\hline & Grade $4-10 \%$ & Grade $4-7 \%$ \\
\hline & Grade $5-1 \%$ & Grade $5-0 \%$ \\
\hline \multirow[t]{5}{*}{$\mathrm{L} 3 / \mathrm{L} 4$} & Grade $1-5 \%$ & Grade $1-3 \%$ \\
\hline & Grade $2-25 \%$ & Grade $2-57 \%$ \\
\hline & Grade $3-45 \%$ & Grade $3-23 \%$ \\
\hline & Grade $4-21 \%$ & Grade $4-16 \%$ \\
\hline & Grade $5-4 \%$ & Grade $5-1 \%$ \\
\hline \multirow[t]{5}{*}{ L4/L5 } & Grade $1-9 \%$ & Grade $1-5 \%$ \\
\hline & Grade $2-21 \%$ & Grade $2-31 \%$ \\
\hline & Grade $3-37 \%$ & Grade $3-43 \%$ \\
\hline & Grade $4-27 \%$ & Grade $4-21 \%$ \\
\hline & Grade $5-6 \%$ & Grade $5-0 \%$ \\
\hline \multirow[t]{5}{*}{ L5/S1 } & Grade $1-6 \%$ & Grade $1-13 \%$ \\
\hline & Grade $2-23 \%$ & Grade $2-22 \%$ \\
\hline & Grade $3-36 \%$ & Grade $3-34 \%$ \\
\hline & Grade $4-22 \%$ & Grade $4-27 \%$ \\
\hline & Grade $5-13 \%$ & Grade $5-4 \%$ \\
\hline
\end{tabular}

were scored as Pfirrmann grades $3-4$ in $86.6 \%$ of cases, Thompson grades 3 as Pfirrmann grades 2-3 in $87.9 \%$ of cases, Thompson grades 2 as Pfirrmann grades $1-2$ in $79.8 \%$ of cases.

A subgroup analysis based on the spinal level revealed weighted Cohen's kappa ranging from 0.40 to 0.70 , with the highest value for $L 5 / S 1$ discs (Table 2).
Percentage agreement ranged from $41 \%$ to $56 \%$, however majority of disagreement occurred due to a one grade difference.

\section{DISCUSSION}

The IVD degeneration is commonly classified using the Pfirrmann grading system when assessed with MRI. There is a scarcity of studies [3] correlating morphological appearance of degeneration with MRI appearance in cadaveric samples. The reliability of the popular Pfirrmann scale has not been comprehensively validated on a large sample using 3 T MRI against the morphological Thompson scale so far. Therefore, the aim of our study was to assess morphological and radiological characteristics of the IVD degeneration and assess the correlation between the Pfirrmann and the Thompson grading systems.

The results of this study showed that overall there is a significant and substantial agreement between morphological and radiological degeneration scales. However, when analysed by IVD levels considerable variability was observed in terms of kappa coefficients, with values as low as 0.4 . Moreover, there was more disagreement in lower grades of degeneration as compared to higher grades, which tended to show more agreement. This suggests better reflection of the stage of degenerative disc disease and as such the clinical applicability of Pfirrmann scale for patients with more degenerated discs. While in vast majority the disagreement between the scales occurred due to a one grade difference, the fact that Pfirrmann scale underscores majority of grades when compared to morphological scale warrants its thoughtful use in a clinical setting. Clinicians should remain careful when following up the patients and relying solely on the descriptions of the MRI exams in the assessment of the progression from lower to higher grades. In such cases, MRI scans should always be evaluated.

The original Pfirrmann grading system was applied to $1 \mathrm{~T} \mathrm{MRI} \mathrm{[9]} \mathrm{and} \mathrm{further} \mathrm{analysed} \mathrm{with} \mathrm{1.5} \mathrm{T} \mathrm{MRI}$

Table 2. Agreement assessment between morphologic Thompson and radiologic Pfirrmann grading systems for degenerative disc disease based on intervertebral disc level

\begin{tabular}{lccc}
\hline Intervertebral disc level & Weighted Cohen's kappa coefficient & P value & Percentage agreement \\
\hline L1/L2 & 0.40 & $<0.05$ & $48.0 \%$ \\
L2/L3 & 0.53 & $<0.001$ & $56.0 \%$ \\
L3/L4 & 0.54 & $<0.001$ & $41.0 \%$ \\
L4/L5 & 0.59 & $<0.001$ & $44.0 \%$ \\
L5/S1 & 0.70 & $<0.001$ & $48.0 \%$ \\
\hline
\end{tabular}


$[6,16]$ as well as with $3 \mathrm{~T}$ and high-resolution $9.4 \mathrm{~T}$ MRI in a pre-clinical research [12]. Our study incorporated $3 \mathrm{~T}$ MRI, which allowed for detailed visualisation of spinal columns. Previous studies have repeatedly shown that T2-signal intensity loss, as one of the few radiological characteristics, is associated with morphologically observed degeneration in cadaveric samples [3, 13]. Moreover, T2-signal intensity correlates strongly with water and proteoglycan content of the disc $[2,18]$, thus its loss should represent the chemical changes that occur within the disc during the degeneration. The T2-signal intensity loss is the main criterion employed in the Pfirrmann scale. Similarly to previous research, the results of this study showed indirectly that the T2-signal intensity loss reflects the process of degeneration, especially for patients with late stages of IVD degeneration.

The use of Thompson grading system in the assessment of the morphology of IVD degeneration has an inherent limitation. The use of only one sagittal section allows only for a limited evaluation of the IVD and might not represent full degree of degeneration throughout the whole IVD. Nonetheless, the midsagittal section provides visualisation of all tissues of the disc structure (NP, AF, endplates, adjacent vertebral bodies) as well as degenerative changes that occur both in coronal and horizontal planes and as such is the most likely to establish the most authentic grade of the degeneration [14]. Moreover, this plane was utilised in the assessment of degeneration using Pfirrmann scale and thus allowed us to directly compare the two grading systems.

The limitation of this study was the use of only male specimens. However, along with the large sample size, this study provides a focused and more representative image of IVD disease for this sex. Further studies should be performed with female patients in order to evaluate any possible sexual dimorphism.

Moreover, the MRI has been performed post-mortem, with absent normal metabolism of tissues. However, only such methodology allows to compare MRI data with full direct macroscopic assessment, and therefore provide reliable and comparable view.

\section{CONCLUSIONS}

With the aging population and with the increase of the prevalence of IVD disease, reliable grading systems of IVD degeneration are crucial for spine surgeons in their clinical assessment. The results of this study showed that overall there was a significant and substantial agreement between radiological Pfirrmann and morphological Thompson grading systems. Nonetheless, clinicians should remain careful when using Pfirrmann scale as the grades tend to deviate from the morphological assessment. Thus, the knowledge of the proper assessment of MRI scans is crucial for spine surgeons.

\section{Acknowledgements}

This research was supported by governmental funds for research in 2016-2019 (Polish Ministry of Science and Higher Education, Diamond Grant, 0182/ /DIA/2016/45). We would like to acknowledge all the donors and their families, whose contribution allowed us to conduct this research.

\section{Conflict of interest: None declared}

\section{REFERENCES}

1. Adams MA, McNally DS, Dolan P. 'Stress' distributions inside intervertebral discs. The effects of age and degeneration. J Bone Joint Surg Br. 1996; 78(6): 965-972, doi: 10.1302/0301-620x78b6.1287, indexed in Pubmed: 8951017.

2. Antoniou J, Steffen T, Nelson F, et al. The human lumbar intervertebral disc: evidence for changes in the biosynthesis and denaturation of the extracellular matrix with growth, maturation, ageing, and degeneration. J Clin Invest. 1996; 98(4): 996-1003, doi: 10.1172/JCl118884, indexed in Pubmed: 8770872.

3. Benneker LM, Heini PF, Anderson SE, et al. Correlation of radiographic and MRI parameters to morphological and biochemical assessment of intervertebral disc degeneration. Eur Spine J. 2005; 14(1): 27-35, doi: 10.1007/s00586004-0759-4, indexed in Pubmed: 15723249.

4. Brinjikji W, Diehn FE, Jarvik JG, et al. MRI findings of disc degeneration are more prevalent in adults with low back pain than in asymptomatic controls: a systematic review and meta-analysis. AJNR Am J Neuroradiol. 2015; 36(12): 2394-2399, doi: 10.3174/ajnr.A4498, indexed in Pubmed: 26359154.

5. GBD 2016 Disease and Injury Incidence and Prevalence Collaborators. Global, regional, and national incidence, prevalence, and years lived with disability for 328 diseases and injuries for 195 countries, 1990-2016: a systematic analysis for the Global Burden of Disease Study 2016. Lancet. 2017; 390(10100): 1211-1259, doi: 10.1016/ S0140-6736(17)32154-2, indexed in Pubmed: 28919117.

6. Griffith JF, Wang YXJ, Antonio GE, et al. Modified Pfirrmann grading system for lumbar intervertebral disc degeneration. Spine (Phila Pa 1976). 2007; 32(24): E708-E712, doi: 10.1097/BRS.0b013e31815a59a0, indexed in Pubmed: 18007231.

7. Martin Bl, Gerkovich MM, Deyo RA, et al. Expenditures and health status among adults with back and neck problems. JAMA. 2008; 299(6): 656-664, doi: 10.1001/ jama.299.6.656, indexed in Pubmed: 18270354. 
8. Modic MT, Steinberg PM, Ross JS, et al. Degenerative disk disease: assessment of changes in vertebral body marrow with MR imaging. Radiology. 1988; 166(1 Pt 1): 193-199, doi: 10.1148/radiology.166.1.3336678, indexed in Pubmed: 3336678.

9. Pfirrmann CW, Metzdorf A, Zanetti M, et al. Magnetic resonance classification of lumbar intervertebral disc degeneration. Spine (Phila Pa 1976). 2001; 26(17): 1873-1878, doi: 10.1097/00007632-200109010-00011, indexed in Pubmed: 11568697.

10. Risbud MV, Shapiro IM. Role of cytokines in intervertebral disc degeneration: pain and disc content. Nat Rev Rheumatol. 2014; 10(1): 44-56, doi: 10.1038/nrrheum.2013.160, indexed in Pubmed: 24166242.

11. Roberts S. Histology and pathology of the human intervertebral disc. J Bone Jt Surg. 2006; 88(2 suppl.): 10.

12. Sher I, Daly C, Oehme D, et al. Novel application of the pfirrmann disc degeneration grading system to $9.4 \mathrm{~T}$ MRI: higher reliability compared to 3T MRI. Spine (Phila Pa 1976). 2019; 44(13): E766-E773, doi: 10.1097/ BRS.0000000000002967, indexed in Pubmed: 31205169.

13. Tertti $M$, Paajanen $H$, Laato $M$, et al. Disc degeneration in magnetic resonance imaging. A comparative biochemical, histologic, and radiologic study in cadaver spines. Spine (Phila Pa 1976). 1991; 16(6): 629-634, doi: 10.1097/00007632-199106000-00006, indexed in Pubmed: 1862401.

14. Thompson JP, Schechter MT, Adams ME, et al. Preliminary evaluation of a scheme for grading the gross morphology of the human intervertebral disc. Spine (Phila Pa 1976). 1990; 15(5): 411-415, doi: 10.1097/00007632199005000-00012, indexed in Pubmed: 2363069.

15. Tomaszewski KA, Saganiak K, Gładysz T, et al. The biology behind the human intervertebral disc and its endplates. Folia Morphol. 2015; 74(2): 157-168, doi: 10.5603/ FM.2015.0026, indexed in Pubmed: 26050801.

16. Urrutia J, Besa P, Campos M, et al. The Pfirrmann classification of lumbar intervertebral disc degeneration: an independent inter- and intra-observer agreement assessment. Eur Spine J. 2016; 25(9): 2728-2733, doi: 10.1007/ s00586-016-4438-z, indexed in Pubmed: 26879918.

17. Vergroesen PPA, Kingma I, Emanuel KS, et al. Mechanics and biology in intervertebral disc degeneration: a vicious circle. Osteoarthritis Cartilage. 2015; 23(7): 1057-1070, doi: 10.1016/j.joca.2015.03.028, indexed in Pubmed: 25827971.

18. Weidenbaum M, Foster RJ, Best BA, et al. Correlating magnetic resonance imaging with the biochemical content of the normal human intervertebral disc. J Orthop Res. 1992; 10(4): 552-561, doi: 10.1002/jor.1100100410, indexed in Pubmed: 1613629. 